\title{
A nested case-control study of influenza vaccination was a cost-effective alternative to a full cohort analysis
}

\author{
E. Hak ${ }^{\mathrm{a}, \mathrm{b}, *}$, F. Wei ${ }^{\mathrm{b}}$, D.E. Grobbee ${ }^{\mathrm{a}}$, K.L. Nichol ${ }^{\mathrm{b}, \mathrm{c}}$ \\ a Julius Center for Health Sciences and Primary Care, University Medical Center Utrecht, PO Box 85060, 3508 AB Utrecht, The Netherlands \\ ${ }^{\mathrm{b}}$ Health Partners Foundation, 8100 34th Ave, Minneapolis, MN 55425, USA \\ ${ }^{\mathrm{c}}$ Veterans Affairs Medical Center, One Veterans Dr, Minneapolis, MN 55417, USA
}

Accepted 30 January 2004

\begin{abstract}
Objective: In the absence of trial results that are applicable to the target population, nested case-control studies might be an alternative to full-cohort analysis. We compared relative and absolute estimates of associations in an influenza vaccine study using both designs.

Study Design and Setting: Data from elderly persons enrolled during six consecutive influenza seasons were used (147,551 personperiods). The endpoints "hospitalization for pneumonia or influenza" (P\&I) or "death" were used combined and separately to define three types of cases. Controls for the case-control samples were randomly selected from the remainder of the total cohort at different ratios (1:1 to 1:4). Logistic regression analysis was used to assess adjusted vaccine effectiveness (VE). Sampling fractions were used to determine the number needed to treat to prevent one outcome. Receiver-operator-curve analysis was conducted to estimate the area under the curve (AUC) as a measure of discriminative capacity of the prognostic model.

Results: In all, 978 P\&I hospitalizations and 1,339 deaths were observed. The adjusted estimates of relative estimates (VE, AUC) and their corresponding $95 \%$ confidence intervals were virtually the same using both study designs, notably when the case-control ratio was high (1:4).

Conclusion: A nested case-control design can provide valid and precise estimates of associations and is a cost-effective alternative for full-cohort analysis. (C) 2004 Elsevier Inc. All rights reserved.
\end{abstract}

Keywords: Influenza; Immunization; Methodology; Case-control study; Cohort study

\section{Introduction}

In the absence of randomized controlled trials (RCT) with clinical endpoints relevant to the individual patient, postmarketing non-experimental studies evaluating the effectiveness or safety of drugs have commonly been applied. This is particularly true for influenza vaccine effectiveness studies [1]. Only one RCT has been conducted on the effectiveness of influenza vaccination in reducing serologically proven influenza among healthy elderly persons [2]. The evidence regarding the vaccine's effectiveness among persons recommended for annual vaccination is derived mainly from non-experimental studies [1]. However, non-experimental designs - mainly cohort and case-control studieshave several advantages over RCTs, including lower cost, enhanced timeliness, larger power, and improved generalizi-

* Corresponding author. Julius Center for Health Sciences and Primary Care, PO Box 85060, 3508 AB, Utrecht The Netherlands. Tel.: +31-302538214; fax: +31-30-2539028.

E-mail address: e.hak@med.uu.nl (E. Hak). bility $[3,4]$. Especially in countries with limited resources to conduct a study, such a design may be attractive. Although prospective cohort studies have consistently been regarded as the strongest among the non-experimental designs $[5,6]$, the case-control approach is more attractive from a logistic and economic point of view, notably when medical records have to be reviewed to retrieve valid information on exposure status or prognostic variables on large numbers of patients [7].

Because large medical databases are available, case-control studies can be conducted while nested in full cohorts. The advantage of a nested case-control design as opposed to the standard case-control approach lies in the absence of bias in the selection of cases or controls; it also allows for directly estimating attributable risks [8-10]. Although much work has been done to prove the similarity of both designs [8-10] and nested case-control studies have been published in this journal $[11,12]$, doubts remain on the validity, precision, and discriminative power of multivariate models in case-control analysis as opposed to full-cohort analysis. We are not aware of any contemporary study evaluating relative and absolute measures when using the two designs. We therefore demonstrate these issues with a recent example 
study within a large cohort of elderly subjects followed prospectively during six consecutive influenza seasons using the HealthPartners computerized influenza vaccination database [13] to compare estimates of influenza vaccine effectiveness, numbers needed to treat and area-under-the-curve (AUC) obtained from case-control analysis with the fullcohort analysis. In the analyses, we further addressed the influence of different case definitions and case-control ratios on the validity and precision of the parameter estimates.

\section{Methods}

\subsection{HealthPartners serial cohort studies: setting and study population}

This study is part of a series of studies on the influenza vaccine effectiveness among elderly members of HealthPartners, Minneapolis-St. Paul, using linked administrative and medical databases [13-15]. Data on more than 20,000 elderly persons in each of the six consecutive influenza seasons during the period 1990 through 1996 with a total of 147,551 person-periods of observation were available for analysis. Details on the setting and collected data have been given in previous reports $[13,14]$. Senior members of the health plan were eligible for inclusion in the yearly cohorts if they were over 65 years of age as of 1 October in the consecutive years, were enrolled continuously throughout the vaccination period (October-December) and influenza season, and if they were noninstitutionalized. The conduct of the study was approved by the medical review boards of the VA Medical Center and HealthPartners.

\subsection{Influenza seasons and endpoints}

Based on surveillance data obtained from the Minnesota Department of Health, each influenza season (1990-1991 through 1995-1996) was defined as occurring between 15 November and 31 March [16]. More than $85 \%$ of the virus isolates was found between these two dates in each of the study years. Except for the first season (1990-1991), which was an influenza type B season, influenza A(H3N1) strains predominated during each of the five subsequent seasons, and influenza activity exceeded threshold levels for two or more consecutive weeks. The endpoints were defined as the occurrence of any hospital admission for pneumonia or influenza (International Classification of Diseases, Ninth revision, Clinical Modification [ICD-9-CM] codes 480-487) and allcause death during the influenza season. These endpoints have shown to be strongly and linearly correlated with influenza virus activity [17], and many studies have shown reductions in the occurrence of these outcomes resulting from the influenza vaccine [18-21].

\subsection{Exposure to influenza vaccination}

For each year, information on the influenza vaccination status before the outcome seasons was retrieved by a computerized search on the code from the Current Procedural Terminology, Fourth Revision (CPT-4) for influenza vaccination
(907.24). From a previous validation study we know that the presence of the codes is in high agreement (93\%) with documentation in the medical records of the patients (K.L. Nichol, unpublished data). There was a good to excellent match between vaccine and circulating virus strains each year except for the 1992-1993 season when the match was moderate (N. Cox, written communication, February 1997). However, in a previous report we have shown that the vaccine also reduced the occurrence of the endpoints under study in that season [13].

\subsection{Covariates}

At baseline, information on the following covariates was available from the medical databases: age, sex, and whether a person was admitted to hospital for influenza and pneumonia or any respiratory condition and the number of outpatient visits in the previous year. Underlying disease of the study subjects was classified into six nonmutually exclusive disease categories according to entries of relevant ICD-9-CM codes in outpatient clinic or hospital databases before the study season: (1) pulmonary disease (ICD-9-CM codes 491496, 500-518), (2) cardiac disease (393-398, 410-414, 425, $428,429)$, (3) diabetes (250), (4) chronic renal disease (581, 582, 585, CPT-4 code 39.95), (5) rheumatologic disease $(446,710,714)$, and (6) dementia or stroke (290-294, 331, $340,341,348,438)$.

\subsection{Nested case-control studies: selection of cases and control subjects}

We used a nested case-control approach to compare parameter estimates with those using the full-cohort database. In the nested case-control study, we defined three types of cases as eligible cohort members who (1) were hospitalized for pneumonia or influenza or died from any cause $(n=$ $1,995)$, (2) were at least once hospitalized for pneumonia or influenza $(n=798)$, or (3) who died $(n=1,339)$ during the influenza epidemics. For each set of the three different cases, one to four times the number of control subjects without an endpoint during either influenza season were randomly sampled using the computerized sampling procedure of SPSS (version 9 for Windows; SPSS Inc, Chicago, IL) referred as samples 1 to 4 . For example, 1,995 subjects were hospitalized or died, and sample 1 included the same number of control subjects $(n=1,995)$, sample 2 included twice the number of control subjects $(n=3,990)$, sample 3 included three times the number of control subjects $(n=5,985)$, and sample 4 included four times the number of control subjects $(n=7,980)$. We present the results of the studies for the three case definitions with these different case-control ratios $(1: 1 ; 1: 2 ; 1: 3 ; 1: 4)$. We estimated the number needed to treat using the sampling fractions. The sampling fractions for sample 1 were 1:73 using the combined endpoint, 1:184 using hospitalization for pneumonia or influenza $(\mathrm{P} \& \mathrm{I})$, and 1:109 using death as the endpoint. The respective other sampling fractions were 1:36, 1:92, and 
$1: 55$ for sample $2 ; 1: 24,1: 61$, and $1: 36$ for sample 3 ; and $1: 18$, 1:46, and 1:27 for sample 4 .

\subsection{Data analysis}

We conducted the data analysis using the aggregated data (unit of analysis is person-period) to enhance the power of the study as previously described [13,14]. Because circulating strains and vaccine compositions differed from season to season, data were assumed to be independent, and, as shown in previous studies, point estimates and standard errors did not change materially after applying techniques taking into account a potential within-person effect. Absence of a characteristic in the medical database was assumed to indicate no presence of the characteristic under study, and therefore missing values were coded absent. Using the fullcohort data and the nested case-control data, univariate analysis with SPSS including Student $t$ tests for continuous and chi-square tests for categorical variables were performed to test for differences between vaccinated and unvaccinated persons. Age was classified into the following five categories according to exponential increase in risk of outcomes: 65 to 69,70 to 74,75 to 79,80 to 89 , and $\geqslant 90$. Similarly, the number of outpatient visits during the previous 12 months was classified into four categories: 0,1 to 6,7 to 12 , and $\geqslant 13$. Next, multivariate logistic regression was applied to assess the association of vaccination status with the endpoints after adjustments for the covariates age, sex, a previous P\&I hospitalization or any hospitalization for a respiratory condition, number of outpatient visits, presence of baseline high-risk disease, and season. The reliability of the multivariate logistic regression model derived from the derivation set was determined by the Hosmer-Lemeshow goodness-of-fit statistic [22]. We calculated odds ratios (ORs) and their 95\% confidence intervals (95\% CIs) as approximation of relative risks. Vaccine effectiveness (VE) was given through the formula 1-OR times $100 \%$ [13].

In the context of non-experimental studies, the numbers needed to treat (i.e., to vaccinate) to prevent one outcome $(\mathrm{NNT})$, given as (1/attibutable risk reduction, ARR) $\times$ 1,000 can be estimated directly only if the average prognosis of treated and nontreated groups is comparable [23,24]. Although the prevalence of some risk factors was somewhat higher in vaccinees than in nonvaccinees, the unadjusted and adjusted estimates of vaccine effectiveness were not materially different. We conservatively used the unadjusted regression weights for vaccination to calculate the NNT values. In a case-control study, the ARR cannot be estimated directly. To give a valid estimate of this risk difference we used the procedure described by Prentice and Pyke [24]. The unadjusted estimate of the regression weight for vaccination was corrected by adding $\log (f)$ to the intercept, where $f$ stands for the fraction of controls sampled from the full cohort. These estimates can be used to estimate the absolute risks of disease in the vaccinated and the nonvaccinated populations and hence the reciprocal of the ARR [25].
Receiver-operator-curves (ROC) and estimates of the area-under-the-curve and their $95 \%$ CIs were calculated to assess the discriminative power of the regression models including vaccination status and the covariates. The area under the ROC can be explained as the probability that the logistic regression model will assign a higher probability of the outcome to a randomly chosen patient with an outcome (hospitalization/death) than to a randomly chosen patient without outcome [26]. An area under the curve (AUC) estimate of 0.5 indicates no discrimination, whereas an estimate of 1.0 indicates perfect discrimination.

\section{Results}

Each season, over 20,000 subjects provided person-periods of observation with a total of 147,551 periods. In all, 1,010 P\&I hospitalizations and 1,339 deaths were recorded during the six seasons. Of the 1,010 hospitalization periods, 212 were second or third occurrences during one season, and we decided to analyze 798 seasonal person-periods with at least one P\&I hospitalization.

Among the noncases of the cohort, the rate was 59.7\%. Because the selection of control subjects was random, exposure rates among control subjects of the case-control samples varied around the same estimate from $58.6 \%$ to $60.8 \%$ using the combined endpoint, from $58.9 \%$ to $60.9 \%$ using P\&I hospitalization, and from $58.1 \%$ to $61.2 \%$ using death as the endpoint. When comparing other baseline data of the unvaccinated and vaccinated control groups, estimations of all covariates were by definition virtually identical as compared with the full cohort analysis independent of the case definition used or the sampling fraction (Table 1).

The overall prediction models for the occurrence of the combined endpoint "any P\&I hospitalization or death from all causes" using the full cohort and the four case-control samples is shown in Table 2. The variables include the vaccine and the covariates age, sex, high-risk disease, any previous hospitalization, number of outpatient visits, and season. The reliability of all models appeared good (HosmerLemeshow Goodness-of-fit test $P>.05$ ). Among all variables in the data set, the presence of high-risk disease (OR $3.28,95 \%$ CI $2.90-3.70$ ), any previous P\&I hospitalization (OR 2.02, 95\% CI 1.77-2.93), and vaccination (OR 0.51, 95\% CI 0.46-0.56) were most strongly associated with the combined endpoint. Except for the 1995-1996 mixed influenza season (influenza $\mathrm{A}[\mathrm{H} 3 \mathrm{~N} 1]$ and influenza $\mathrm{A}[\mathrm{H} 1 \mathrm{~N} 1]$ ), the presence of a mono-influenza $\mathrm{A}(\mathrm{H} 3 \mathrm{~N} 1)$-type epidemic substantially increased the risk of developing complications as compared with the first influenza B-type season. There were no major differences in the relative risk estimates and the corresponding $95 \%$ CIs between the full cohort analysis and the nested case-control samples.

Table 3 shows the estimates of vaccine effectiveness, NNT, and the area under the ROC and their corresponding 95\% CIs using the same fitted regression model for each 
Table 1

Influence of case-control sampling on distribution of risk factors in unvaccinated (Vac-) and vaccinated (Vac+) persons estimated from control subjects

\begin{tabular}{|c|c|c|c|c|c|c|c|c|c|c|}
\hline \multirow[b]{2}{*}{ Study sample } & \multicolumn{2}{|c|}{ Age mean (SD) } & \multicolumn{2}{|c|}{ Male $(\%)$} & \multicolumn{2}{|c|}{ High-risk $^{\mathrm{a}}(\%)$} & \multicolumn{2}{|c|}{ Hospitalization $^{\mathrm{b}}(\%)$} & \multicolumn{2}{|c|}{ Outpatient visits, mean (SD) } \\
\hline & $\mathrm{Vac}-$ & $\mathrm{Vac}+$ & $\mathrm{Vac}-$ & $\mathrm{Vac}+$ & $\mathrm{Vac}-$ & $\mathrm{Vac}+$ & $\mathrm{Vac}-$ & $\mathrm{Vac}+$ & $\mathrm{Vac}-$ & $\mathrm{Vac}+$ \\
\hline Cohort & $72.6(6.6)$ & $72.5(5.6)$ & 40.8 & 44.6 & 29.4 & 42.3 & 11.4 & 14.0 & $8.9(11.7)$ & $12.9(11.6)$ \\
\hline \multicolumn{11}{|l|}{ Hospitalization/death } \\
\hline Sample $1^{\mathrm{c}}$ & $72.6(6.6)$ & $72.8(5.8)$ & 37.6 & 46.2 & 29.6 & 42.8 & 14.8 & 16.7 & $9.1(12.1)$ & $13.3(11.0)$ \\
\hline Sample 2 & $72.3(6.5)$ & $72.7(5.8)$ & 40.2 & 46.5 & 30.4 & 44.0 & 10.5 & 14.8 & $8.8(11.9)$ & $13.1(11.8)$ \\
\hline Sample 3 & $72.6(6.5)$ & $72.5(5.7)$ & 40.9 & 45.3 & 29.3 & 41.8 & 11.7 & 13.5 & $8.7(11.5)$ & $13.1(11.7)$ \\
\hline Sample 4 & $72.6(6.7)$ & $72.4(5.6)$ & 40.4 & 45.5 & 29.4 & 41.5 & 11.5 & 13.1 & $8.6(11.2)$ & $12.9(11.4)$ \\
\hline \multicolumn{11}{|l|}{ Hospitalization } \\
\hline Sample 1 & $72.3(6.7)$ & $72.5(5.9)$ & 39.4 & 41.4 & 29.8 & 43.0 & 11.9 & 14.8 & $9.3(12.7)$ & $12.6(11.3)$ \\
\hline Sample 2 & $72.2(6.8)$ & $72.2(5.5)$ & 44.2 & 44.1 & 34.5 & 44.1 & 10.8 & 13.9 & $8.6(10.3)$ & $12.8(11.7)$ \\
\hline Sample 3 & $72.6(6.6)$ & $72.5(5.6)$ & 40.3 & 43.5 & 27.8 & 42.8 & 11.3 & 14.1 & $8.9(12.3)$ & $12.9(12.0)$ \\
\hline Sample 4 & $72.5(6.5)$ & $72.6(5.6)$ & 42.6 & 45.3 & 29.6 & 42.5 & 11.7 & 13.5 & $9.1(12.8)$ & $12.9(11.6)$ \\
\hline \multicolumn{11}{|l|}{ Death } \\
\hline Sample 1 & $72.9(6.8)$ & $72.4(5.6)$ & 41.7 & 45.9 & 30.8 & 41.9 & 13.0 & 14.1 & $9.3(16.0)$ & $12.6(11.7)$ \\
\hline Sample 2 & $72.6(6.8)$ & $72.7(5.5)$ & 40.0 & 43.4 & 29.7 & 41.9 & 8.9 & 14.3 & $8.5(11.5)$ & $12.8(11.5)$ \\
\hline Sample 3 & $72.9(6.8)$ & $72.6(6.0)$ & 38.6 & 43.9 & 28.9 & 42.3 & 11.8 & 13.2 & $8.9(11.5)$ & $13.0(12.7)$ \\
\hline Sample 4 & $72.5(6.5)$ & $72.4(5.5)$ & 40.5 & 43.8 & 30.1 & 42.0 & 11.1 & 15.0 & $8.9(13.2)$ & 12.9 \\
\hline
\end{tabular}

${ }^{a}$ High-risk denotes having at least one of the following high-risk diseases: heart disease, lung disease, diabetes mellitus, renal disease, rheumatologic disease, or dementia/stroke.

b Hospitalization for any chronic respiratory condition in 12 months.

c Sample 1 to 4 refer to the case-control ratios: 1:1, 1:2, 1:3, and 1:4.

study sample. In the full-cohort analysis, the vaccine effectiveness seemed to be $49 \%$ (95\% CI 44-54) in preventing P\&I hospitalization or death, 32\% (95\% CI 21-41) in preventing P\&I hospitalizations, and 57\% (95\% CI 51-61) in reducing deaths from any cause after adjustments for the baseline characteristics. The estimates of vaccine estimates in reducing the occurrence of the combined outcome, P\&I hospitalization, or death using the case-control samples varied within a small range from $46 \%$ to $52 \%, 24 \%$ to $38 \%$, and $51 \%$ to $60 \%$, respectively, with CIs largely overlapping.

The NNTs were 159, 1,016, and 174 for the combined endpoint, P\&I hospitalization, or death, respectively, in the full-cohort analysis. Point estimates were somewhat different for the smaller case-control samples and tended to underestimate the NNT.

The discriminative power of the multivariate models, all with reliable fit (goodness-of-fit test $P>.05$ ), was high (AUC estimates between 0.77 and 0.82 ), and there were no substantial differences between the two designs.

\section{Discussion}

Our study illustrates vaccine effectiveness study that supports earlier observations on other drugs showing that a nested case-control approach provides similar estimates of

Table 2

Relative risk estimates for the combined endpoint based on full-cohort and case-control sampling ${ }^{\mathrm{a}}$

\begin{tabular}{|c|c|c|c|c|c|}
\hline Variable & Full cohort $(n=147,551)^{\mathrm{b}}$ & Sample $1(n=3,990)$ & Sample $2(n=5,985)$ & Sample $3(n=7,980)$ & Sample $4(n=9,975)$ \\
\hline Vaccine & $0.51(0.46-0.56)$ & $0.54(0.47-0.63)$ & $0.48(0.42-0.55)$ & $0.54(0.48-0.61)$ & $0.52(0.46-0.58)$ \\
\hline Age, $\mathrm{yr}^{\mathrm{c}}$ & $1.51(1.45-1.57)$ & $1.55(1.46-1.65)$ & $1.56(1.48-1.64)$ & $1.55(1.48-1.63)$ & $1.54(1.47-1.61)$ \\
\hline Male & $1.53(1.40-1.67)$ & $1.71(1.48-1.99)$ & $1.46(1.29-1.66)$ & $1.52(1.35-1.70)$ & $1.55(1.39-1.74)$ \\
\hline High-risk disease $^{\mathrm{d}}$ & $3.28(2.90-3.70)$ & $3.61(3.04-4.29)$ & $3.05(2.63-3.53)$ & $3.45(3.00-3.97)$ & $3.31(2.88-3.79)$ \\
\hline Any CRD hospitalization & $1.71(1.54-1.90)$ & $1.39(1.15-1.67)$ & $1.77(1.52-2.07)$ & $1.76(1.53-2.03)$ & $1.81(1.58-2.08)$ \\
\hline Any P\&I hospitalization & $2.02(1.77-2.31)$ & $2.24(1.66-2.99)$ & $2.38(1.87-3.02)$ & $2.41(1.94-2.99)$ & $2.34(1.92-2.86)$ \\
\hline Outpatient visits & $1.22(1.15-1.30)$ & $1.18(1.08-1.29)$ & $1.24(1.15-1.34)$ & $1.18(1.10-1.27)$ & $1.21(1.13-130)$ \\
\hline Season $2^{\mathrm{f}}$ & $1.72(1.46-2.03)$ & $1.69(1.31-2.18)$ & $1.56(1.25-1.99)$ & $1.65(1.34-2.03)$ & $1.76(1.44-2.15)$ \\
\hline Season 3 & $1.29(1.08-1.53)$ & $1.27(0.98-1.64)$ & $1.20(0.96-1.50)$ & $1.22(0.99-1.51)$ & $1.30(1.06-1.60)$ \\
\hline Season 4 & $1.71(1.45-2.03)$ & $1.65(1.27-2.13)$ & $1.60(1.28-2.00)$ & $1.83(1.48-2.26)$ & $1.69(1.38-2.06)$ \\
\hline Season 5 & $1.72(1.45-2.03)$ & $1.67(1.29-2.17)$ & $1.64(1.31-2.06)$ & $1.71(1.38-2.12)$ & $1.69(1.38-2.08)$ \\
\hline Season 6 & $1.07(0.88-1.28)$ & $0.88(0.67-1.17)$ & $0.93(0.73-1.19)$ & $0.97(0.77-1.22)$ & $1.06(0.85-1.32)$ \\
\hline
\end{tabular}

${ }^{a}$ Goodness of fit $P$ value, full model: .29; sample 1: .71; sample 2: .19; sample 3: .29; sample 4: .47.

b Values are adjusted odds ratios with $95 \%$ confidence intervals in parentheses.

c Odds ratio is given for the categorized variable: $65-69,70-74,75-79,80-89$, and $\geqslant 90$ years.

${ }^{\mathrm{d}}$ High-risk disease denoted the presence of at least one of the following conditions: heart disease, lung disease, diabetes, renal disease, rheumatologic disease, or stroke/dementia.

${ }^{\mathrm{e}}$ Given for the categorized variable: $0,1-6,7-12, \geqslant 13$.

${ }^{\mathrm{f}}$ Reference is year 1 . 
Table 3

Vaccine effectiveness (VE), number needed to vaccinate to prevent one outcome (NNT), and area-under-the-curve (AUC) in full-cohort and nested case-control samples

\begin{tabular}{|c|c|c|c|c|c|c|}
\hline Populations & Total $(n)$ & Cases $(n)$ & Controls $(n)$ & $\operatorname{VE}(95 \% \mathrm{CI})^{\mathrm{a}}$ & $\mathrm{NNT}^{\mathrm{b}}$ & $\operatorname{AUC}(95 \% \mathrm{CI})$ \\
\hline \multicolumn{7}{|c|}{ P\&I hospitalization/death } \\
\hline Cohort & 147,551 & 1,995 & 145,556 & $49(44-54)$ & 159 & $0.80(0.79-0.81)$ \\
\hline Sample $1^{\mathrm{c}}$ & 3,990 & 1,995 & 1,995 & $46(37-53)$ & 179 & $0.79(0.78-0.80)$ \\
\hline Sample 2 & 5,985 & 1,995 & 3,990 & $52(45-58)$ & 143 & $0.79(0.78-0.81)$ \\
\hline Sample 3 & 7,980 & 1,995 & 5,985 & $46(39-52)$ & 167 & $0.80(0.79-0.81)$ \\
\hline Sample 4 & 9,975 & 1,995 & 7,980 & $48(42-54)$ & 157 & $0.80(0.79-0.81)$ \\
\hline \multicolumn{7}{|c|}{ P\&I hospitalisation } \\
\hline Cohort & 147,551 & 798 & 146,753 & $32(21-41)$ & 1016 & $0.77(0.76-0.79)$ \\
\hline Sample 1 & 1,596 & 798 & 798 & $38(21-51)$ & 795 & $0.77(0.75-0.80)$ \\
\hline Sample 2 & 2,394 & 798 & 1,596 & $24(7-38)$ & 1228 & $0.77(0.75-0.79)$ \\
\hline Sample 3 & 3,192 & 798 & 3,192 & $35(21-46)$ & 1007 & $0.77(0.75-0.79)$ \\
\hline Sample 4 & 3,990 & 798 & 3,990 & $34(21-45)$ & 830 & $0.77(0.76-0.79)$ \\
\hline \multicolumn{7}{|l|}{ Death } \\
\hline Cohort & 147,551 & 1,339 & 146,212 & $57(51-61)$ & 174 & $0.82(0.80-0.83)$ \\
\hline Sample 1 & 2,678 & 1,339 & 1,339 & $51(40-59)$ & 194 & $0.81(0.79-0.82)$ \\
\hline Sample 2 & 4,017 & 1,339 & 2,678 & $60(52-66)$ & 157 & $0.82(0.80-0.83)$ \\
\hline Sample 3 & 5,356 & 1,339 & 4,017 & $59(42-64)$ & 161 & $0.82(0.80-0.83)$ \\
\hline Sample 4 & 6,695 & 1,339 & 5,356 & $57(50-63)$ & 182 & $0.82(0.80-0.83)$ \\
\hline
\end{tabular}

vaccine effectiveness, NNT, and AUC independent of the case definition or the sampling fraction used as opposed to the full-cohort analysis. Therefore, a nested case-control design provides a cost-effective alternative for the non-experimental evaluation of drug effects using a full-cohort design.

To appreciate our findings, some characteristics of our study need to be addressed. Because all information from regular outpatient visits and hospitalizations were routinely entered in databases independent of any research protocols, the information on endpoints in the total cohort and casecontrol samples was independent of the exposure status. The exposure rates and distribution of prevalence of some important risk factors across the exposure groups did not substantially differ in control subjects using the cohort data or the nested case-control sampling, and by definition selection bias is absent in the nested case-control samples. This was illustrated by similar distributions of exposure rates and various covariates in the full cohort and control samples. However, confounding bias or incomparability of exposure groups is a potential threat to the validity of the study [27]. Some major risk factors were more common in the vaccinated than in the unvaccinated persons. However, because we were able to capture data on several important prognostic variables and because adjustment for these in the multivariate analysis led to minor changes in the estimates, it is unlikely that confounding explains our findings.

The relative risks of each variable and its corresponding 95\% CIs were similar when comparing the case-control samples with the full-cohort analysis (see Table 2). Our observation that the presence of high-risk disease, previous hospitalization for P\&I, and vaccination were the strongest predictors for the combined endpoint agrees with previous findings $[18,21]$. Because this was a serial study covering six different influenza seasons, we were able to demonstrate the influence of circulating strains on the occurrence of serious outcomes. There seemed to be a strong association (OR between 1.3 and 1.7) between the occurrence of a mono influenza $\mathrm{A}(\mathrm{H} 3 \mathrm{~N} 1)$ epidemic and the incidence of complications.

Vaccination seemed to reduce the incidence of P\&I hospitalization or death by $49 \%$, hospitalization by $32 \%$, and death by $57 \%$. These findings agree with a recent large study among elderly members of three HMOs followed during the 1996/97 and 1997/98 influenza A epidemics [28]. The estimate of 159 persons that need to be vaccinated to prevent one serious outcome was somewhat higher than in the large HMO study (102 persons). The relative reduction in these severe complications agreed with the effect estimates as observed in the trial by Govaert et al. [2]. We may therefore claim that non-experimental designs can give valid estimates of vaccine effectiveness as compared with RCTs. Non-experimental studies can be designed to study more severe outcomes from influenza, such as respiratory and cardiovascular disease or death. Such outcomes can be studied only in trials with several thousands of participants, which makes such a trial costly and unfeasible. In addition, the study population from medical databases might be more representative for the target population for influenza vaccination; hence, the applicability of the results for daily practice can be enhanced. Finally, case-control studies are designed more efficiently, leading to reductions in costs. It is important for countries 
worldwide to use such study designs to show vaccine effectiveness provided they allow for control for confounding by indication [27].

For medical decision-making, clinical prediction rules can be used by physicians and patients to identify high- and low-risk persons on the basis of available prognostic patient information [29]. One measure to quantify the discriminative power of the multivariate is the area-under-the-receiver-operating-curve (AUC) estimate. In all study samples the AUC estimates were high, ranging from 0.76 to 0.81 , which enables the development of a rule with accurate test properties. In the previously mentioned large HMO study we developed a prediction rule on the basis of similar prognostic information as in this study, and the AUC was similar (AUC 0.84). The rule seemed to have accurate predictive properties to discriminate between high-risk and low-risk persons. The present data demonstrate that nested case-control studies might also be used to develop such a prediction rule with similar discriminative capacity as the full-cohort analysis. For the development of the quantitative rule, the prevalence of disease in the total cohort estimated by use of the intercept of the regression equation needs to be corrected as proposed by Prentice and Pike [24].

In conclusion, randomized trials may not be needed to estimate the vaccine effectiveness for the target population of influenza vaccination. By using a non-experimental approach, we demonstrated that nested case-control studies provide cost-effective alternatives for full-cohort analyses, and such a design should therefore receive more attention in the evaluation of vaccines and other therapeutic or preventive drugs.

\section{References}

[1] Hak E, Hoes AW, Verheij TJM. Influenza vaccination: who needs them and when? Drugs 2002;62:2413-20.

[2] Govaert Govaert ThME, Thijs CTMCN , Masurel N, Sprenger MJW, Dinant GJ, Knottnerus JA. The efficacy of influenza vaccination in elderly individuals: a randomized double-blind placebo-controlled trial. JAMA 1994;272:1661-5.

[3] Benson K, Hartz A. A comparison of observational studies and randomized, controlled trials. N Engl J Med 2000;342:1878-86.

[4] Concato J, Shah N, Horwitz RI. Randomized, controlled trials, observational studies, and the hierarchy of research designs. N Engl J Med 2000;342:1887-92.

[5] Sacket DL, Haynes RB, Tugwell P. Clinical epidemiology: a basic science for clinical medicine. Boston: Little, Brown; 1985.

[6] Austin H, Hill HA, Flanders D, Greeneberg RS. Limitations in the application of case-control methodology. Epidemiol Rev 1994;16: 65-76.

[7] Hak E, Hoes AW, Grobbee DE, Lammers JWJ, Van Essen GA, Van Loon AM, Verheij TJM. Conventional influenza vaccination is not associated with severe complications among adults with chronic pulmonary disease. Am J Epidemiol 2003;157:692-700.

[8] Mietinen OS. Estimability and estimation in case-referent studies. Am J Epidemiol 1976;103:181-235.
[9] Freeman J, Hutchison GB. Prevalence, incidence and duration. Am J Epidemiol 1980;112:707-23.

[10] Breslow NE. Statistics in epidemiology: the case-control study. J Am Stat Assoc 1996;91:14-28.

[11] Concato J, Peduzzi P, Kamina A, Horwitz RI. A nested case-control study of the effectiveness of screening for prostate cancer: research design. J Clin Epidemiol 2001;54:558-64.

[12] Lagnaoui R, Begaud B, Moore N, Chaslerie A, Fourrier A, Letenneur L, Dartiques J, Moride Y. Benzodiazepine use and risk of dementia: a nested case-control study. J Clin Epidemiol 2002;55:314-8.

[13] Nichol KL, Wuorenma J, Von Sternberg T. Benefits of influenza vaccination for low-, intermediate- and high-risk senior citizens. Arch Intern Med 1998;158:1769-76.

[14] Nichol KL, Margolis KL, Wuorenma J, Von Sternberg T. The efficacy and cost-effectiveness of vaccination against influenza among elderly persons living in the community. N Engl J Med 1994;331: 778-784.

[15] Nichol KL, Baken L, Nelson A. Relation between influenza vaccination and outpatient visits, hospitalization, and mortality in elderly persons with chronic lung disease. Ann Intern Med 1999;130:397403.

[16] Minnesota department of Health. Annual summary of communicable diseases reported to the Minnesota Department of health. 1995. Dis Control Newsletter 1995;23:32-40.

[17] Simonsen L, Fukuda K, Schonberger LB, Cox NJ. The impact of influenza epidemics on hospitalizations. J Infect Dis 2000;181:831-7.

[18] Ahmed AH, Nicholson KG, Nguyen-Van-tam JS. Reduction in mortality associated with influenza vaccine during 1989-90 epidemic. Lancet 1995;346:591-5.

[19] Fedson DS, Wadja A, Nicol JP, Hammond GW, Kaiser DL, Roos LL. Clinical effectiveness of influenza vaccination in Manitoba. JAMA 1993;270:1956-61.

[20] Ohmit SE, Monto AS. Influenza vaccine effectiveness in preventing hospitalization for pneumonia among the elderly during influenza A and type B seasons. Int J Epidemiol 1995;24:1240-8.

[21] Fleming DM, Watson JM, Nicholas S, Smith GE, Swan AV. Study on the effectiveness of influenza vaccination in the elderly in the epidemic of 1989-90 using a general practice database. Epidemiol Infect 1995;115:581-9.

[22] Hosmer DW, Lemeshow S. Eds. Assessing the fit of the model. In: Applied logistic regression. New York: J. Wiley; 1989. p. 135-75.

[23] Lubsen K, Hoes AW, Grobbee DE. Implications of trial results: the potentially misleading notion of number needed to treat and average duration of life. Lancet 2000;356:1757-9.

[24] Prentice RL, Pyke R. Logistic disease incidence models and casecontrol studies. Biometrika 1979;66:403-11.

[25] Altman DG. Confidence intervals for the number needed to treat. BMJ 1998;317:1309-12.

[26] Hanley JA, McNeill BJ. The meaning and use of the area under a receiver operating characteristic curve (ROC). Radiology 1982;143: 29-36.

[27] Hak E, Verheij TJM, Grobbee DE, Nichol KL, Hoes AW. Confounding by indication in non-experimental evaluation of vaccine effectiveness: the example of prevention of influenza complications. J Epidemiol Community Health 2002;56:951-5.

[28] Nordin J, Mullooly J, Poblete S, Strikas R, Petrucci R, Wei F, Rush B, Safirstein B, Wheeler D, Nichol KL. Influenza vaccine effectiveness in preventing hospitalizations and deaths in persons 65 years or older in Minnesota, New York and Oregon: data from 3 health plans. J Infect Dis 2001;184:665-70.

[29] Hak E, Wei F, Nordin J, Mullooly J, Poblete S, Nichol KL. A clinical prediction rule for pneumonia and influenza hospitalization and death during influenza epidemics. J Infect Dis 2004;189:450-8. 